\title{
Adrenal Gland Neuroblastoma
}

National Cancer Institute

\section{Source}

National Cancer Institute. Adrenal Gland Neuroblastoma. NCI Thesaurus. Code C4827.

A neuroblastoma arising from the adrenal gland. 